Parallel Processing Letters, Vol. 12, Nos. 3 \& 4 (2002) 385-386

(C) World Scientific Publishing Company

\title{
AUTHOR INDEX \\ VOLUME 12 (2002)
}

Afsahi, A. and Dimopoulos, N. J., Analysis of a latency hiding broadcasting algorithm on a reconfigurable optica interconnect

Aguilar, J., A graph theoretical model for scheduling simultaneous I/O operations on parallel and distributed environments

12 (2002) 41-50

Alima, L. O., see Johnen, C.

Alt, M., Bischof, H. and Gorlatch, S., Program development for computational grids using skeletons and performance prediction

12 (2002) 157-174

Barth, D. and Fragopoulou, P., Compact multicast algorithms on grids and tori without intermediate buffering

12 (2002) 31-39

Beauquier, B. and Darrot, E., On arbitrary size Waksman networks and their vulnerability

Bischof, H., see Alt, M.

Chakravarty, M. M. T., see Lechtchinsky, R.

Chong, F. T., see Oskin, M.

Cole, M., see Hayashi, Y.

Cole, M., see Kuchen, H.

Darrot, E., see Beauquier, B.

Das, S. K., see Panda, B. S.

Datta, A. K., see Johnen, C.

Dimopoulos, N. J., see Afsahi, A.

Dobrev, S. and Vrťo, I., Optimal broadcasting in tori with dynamic faults

Fischer, J. and Gorlatch, S., Turing universality of recursive patterns for parallel programming

Fragopoulou, P., see Barth, D.

Gerlach, J., Generic programming of parallel applications with Janus

Gorlatch, S. and Lengauer, C., Foreword

Gorlatch, S., see Alt, M.

Gorlatch, S., see Fischer, J.

Ha, S., see Kee, Y.

Hayashi, Y. and Cole, M., Automated cost analysis of a parallel maximum segment sum program derivation

Hensley, J., see Oskin, M.

Herrmann, C. A. and Lengauer, C., Using metaprogramming to parallelize functional specifications

12 (2002) $113-125$

12 (2002) 327-340

12 (2002) 287-296

12 (2002) $157-174$

12 (2002) 249-266

12 (2002) 311-326

12 (2002) 95-111

12 (2002) 141-155

12 (2002) 287-296

12 (2002) 51-64

12 (2002) 327-340

12 (2002) 41-50

12 (2002) 17-22

12 (2002) 229-246

12 (2002) 31-39

12 (2002) 175-190

12 (2002) 137

12 (2002) 157-174

12 (2002) 229-246

12 (2002) 65-77

12 (2002) 95-111

12 (2002) 311-326

12 (2002) 193-210

Hidalgo-Herrero, M. and Ortega-Mallén, Y., An operational semantics for the parallel language Eden

12 (2002) 211-228 
Hsu, W. J., see Loh, P. K. K.

Irony, D. and Toledo, S., Trading replication for communication in parallel distributed-memory dense solvers

Johnen, C., Alima, L. O., Datta, A. K. and Tixeuil, S., Optimal snap-stabilizing neighborhood synchronizer in tree networks

Kavi, K. M. and Mehta, D. P., Mutual exclusion on optical buses

Kee, Y. and Ha, S., An efficient implementation of the BSP programming library for VIA

Keen, D., see Oskin, M.

Keller, G., see Lechtchinsky, R.

Kuchen, H. and Cole, M., The integration of task and data parallel skeletons

Lavault, C., Embeddings into the pancake interconnection network

Lechtchinsky, R., Chakravarty, M. M. T. and Keller, G., Costing nested array codes

Lengauer, C., see Gorlatch, S.

Lengauer, C., see Herrmann, C. A.

Lita, L.-V., see Oskin, M.

Loh, P. K. K. and Hsu, W. J., Embedding of fault-tolerant trees in the Josephus cube

Mehta, D. P., see Kavi, K. M.

Natarajan, V., see Panda, B. S.

Nitsche, T., Lifting sequential functions to parallel skeletons

Ortega-Mallén, Y., see Hidalgo-Herrero, M.

Oskin, M., Keen, D., Hensley, J., Lita, L.-V. and Chong, F. T., Operating systems techniques for parallel computation in intelligent memory

Panda, B. S., Natarajan, V. and Das, S. K., Parallel algorithms for Hamiltonian 2-separator chordal graphs

Paterson, M. S., Schröder, H., Sýkora, O. and Vrťo, I., Permutation communication in all-optical rings

Rajasekaran, S., Efficient parallel algorithms for template matching

Sýkora, O., see Paterson, M. S.

Schröder, H., see Paterson, M. S.

Serna, M. and Xhafa, F., The parallel approximability of the false and true gates problems for NOR-circuits

Sibeyn, J. F., Optimal list ranking on one-dimensional arrays

Tixeuil, S., see Johnen, C.

Toledo, S., see Irony, D.

Ushijima, K., see Xiang, L.

Vrťo, I., see Dobrev, S.

Vrťo, I., see Paterson, M. S.

Xhafa, F., see Serna, M.

Xiang, L., Ushijima, K. and Zhao, J., Time optimal $n$-size matching parentheses and binary tree decoding algorithms on a $p$-processor BSR

Zhao, J., see Xiang, L.

\author{
$12(2002) 3-16$ \\ $12(2002) 79-94$ \\ 12 (2002) 327-340 \\ 12 (2002) 341-358 \\ 12 (2002) 65-77 \\ 12 (2002) 311-326 \\ 12 (2002) 249-266 \\ 12 (2002) 141-155 \\ 12 (2002) 297-310 \\ 12 (2002) 249-266 \\ 12 (2002) 137 \\ 12 (2002) 193-210 \\ 12 (2002) 311-326 \\ 12 (2002) 3-16 \\ 12 (2002) 341-358 \\ 12 (2002) 51-64 \\ 12 (2002) 267-284 \\ 12 (2002) 211-228
}

12 (2002) 311-326

12 (2002) 51-64

12 (2002) 23-29

12 (2002) 359-364

12 (2002) 23-29

12 (2002) 23-29

12 (2002) 127-136

12 (2002) 375-383

12 (2002) 327-340

12 (2002) 79-94

12 (2002) 365-374

12 (2002) 17-22

12 (2002) 23-29

12 (2002) 127-136

12 (2002) 365-374

12 (2002) 365-374 